\title{
Semi-comprehensive analysis of gene amplification in gastric cancers using multiplex ligation-dependent probe amplification and fluorescence in situ hybridization
}

\author{
Akishi Ooi ${ }^{1,2}$, Takeru Oyama ${ }^{1}$, Ritsuko Nakamura ${ }^{1}$, Ryosuke Tajiri ${ }^{1}$, Hiroko Ikeda ${ }^{2}$, \\ Sachio Fushida ${ }^{3}$, Hiroyuki Nakamura ${ }^{4}$ and Yoh Dobashi ${ }^{5}$ \\ ${ }^{1}$ Department of Molecular and Cellular Pathology, Graduate School of Medical Science, Kanazawa University, \\ Ishikawa, Japan; ${ }^{2}$ Pathology Section, University Hospital, Kanazawa University, Ishikawa, Japan; ${ }^{3}$ Department \\ of Surgery, Graduate School of Medical Science, Kanazawa University, Ishikawa, Japan; ${ }^{4}$ Department of \\ Environmental and Preventive Medicine, Graduate School of Medical Science, Kanazawa University, Ishikawa, \\ Japan and ${ }^{5}$ Department of Pathology, Saitama Medical Center, Jichi Medical University, Saitama, Japan
}

The prognosis of patients with gastric carcinomas at an advanced stage still remains dismal, and therefore novel therapeutic modalities are urgently needed. Since the successful targeting of amplified ERBB2 with a humanized monoclonal antibody, the amplified genes of other receptor tyrosine kinases such as EGFR, FGFR2, and MET, as well as those of other cell regulator genes, are being considered as candidate targets of molecular therapy. The aim of the present study was to determine the amplification status of 26 genes, which are frequently amplified in solid cancers, in advanced gastric cancers. A total of 93 formalin-fixed and paraffin-embedded advanced gastric cancer tissues were examined by multiple ligation-dependent probe amplification, and 32 cases with 'gain' or 'amplified' status of $\mathbf{1 6}$ genes were further examined for the respective gene amplification by fluorescence in situ hybridization (FISH) and for the respective protein overexpression by immunohistochemistry. The frequencies of gene amplifications in advanced gastric cancers were as follows: ERBB2 (13 cases, 14\%), FGFR2 (7 cases, $8 \%$ ), MYC (7 cases, 8\%), TOP2A ( 7 cases, $8 \%$ ), MET (4 cases, $4 \%$ ), MDM2 (4 cases, $4 \%$ ), CCND1 (3 cases, $3 \%)$, FGF10 ( 2 cases, 3\%), and EGFR (1 case, 1\%). Amplification of the receptor tyrosine kinases genes occurred in a mutually exclusive manner except for one tumor in which ERBB2 and FGFR2 were both amplified but in different cancer cells. Co-amplification of ERBB2 and MYC, and EGFR and CCND1, in single nuclei but on different amplicons, was confirmed in one case each. Attempts at correlating the FISH status with the immunohistochemical staining pattern showed variable results from complete concordance to no correlation. In conclusion, combination of multiple ligation-dependent probe amplification and FISH analysis is a feasible approach for obtaining the semi-comprehensive genetic information that is necessary for personalized molecular targeted therapy.

Modern Pathology (2015) 28, 861-871; doi:10.1038/modpathol.2015.33; published online 6 March 2015

Gastric cancer is the fourth most common malignancy and ranks second among all cancer deaths worldwide. ${ }^{1}$ According to the Japanese Research Society for Gastric Cancer, gastric cancers are divided into two groups: early gastric cancers that are confined to the mucosa and submucosa, and

Correspondence: Professor A Ooi, MD, PhD, Department of Molecular and Cellular Pathology Graduate School of Medical Science, Kanazawa University, Ishikawa 920-8641, Japan.

E-mail: aooi@med.kanazawa-u.ac.jp

Received 2 October 2014; revised 13 December 2014; accepted 15

December 2014; published online 6 March 2015 advanced gastric cancers that penetrate the muscle layer or beyond. ${ }^{2}$ Estimated proportions of surviving Japanese patients with 'early gastric cancer' and 'advanced gastric cancer' in the 5-years after surgery are 98.5 and $51.7 \%$, respectively. ${ }^{3}$ Thus, novel therapeutic modalities are urgently needed for the treatment of advanced gastric carcinomas.

In 2009, an open-label, international phase 3 , randomized controlled trial (ToGA, Trastuzumab for gastric cancer) found that the addition of trastuzumab, a humanized monoclonal antibody that binds to the extracellular juxtamembrane domain of a receptor tyrosine kinase, ERBB2, (otherwise known 
as the human epidermal growth factor receptor 2), to cisplatin-based chemotherapy significantly improved the overall survival of advanced gastric cancer patients with overexpressed and/or amplified ERBB2 as compared with chemotherapy alone. ${ }^{4}$ To date, targeting ERBB2-overexpression/amplification remains the only successful and approved targeted therapy for gastric cancer. However, there is an urgent need for other inhibitors to be investigated for the therapy of many of the unique molecular subtypes of gastric cancer. ${ }^{5}$ In particular, FGFR2 and $M E T$, which encode RTKs similar to ERBB2, are considered as strong candidate targets because their amplification is associated with advanced stages, ${ }^{6,7}$ and furthermore, the amplification of FGFR2 was preferentially found in a poorly differentiated subtype. ${ }^{8,9}$ In preclinical studies, cells with amplified FGFR2 or MET showed overexpression of their respective proteins, and inhibitors to these receptors were shown to effectively block their downstream signal transduction and induce apoptosis. ${ }^{10-14}$ At present, apart from ERBB2 targeting, approaches for targeting MET and FGFR2 are the most clinically advanced of prospective targeted therapy. However, the prevalence of amplification of each of these genes, as assessed by oligonucleotide array comparative genetic hybridization, real-time PCR, fluorescence in situ hybridization (FISH), or silver in situ hybridization is low, ie, from 2 to $5 \%$ for FGFR2, ${ }^{9,10,12,14}$ and from 2 to $8 \%$ for $M E T$, respectively. ${ }^{6,7,14,15}$ Intriguingly, a recent comprehensive genome-wide analysis of copy number alterations using an single nucleotide polymorphism array ${ }^{13}$ showed that amplification of FGFR2, EGFR, $E R B B 2$, and $M E T$ occurred mutually exclusively and that other genes such as $M Y C$ and CCND1 were also amplified in gastric cancer. When considering promising targets of molecular therapy, the apparently low prevalence of these gene amplifications in gastric cancer makes selection of the right set of patients that can benefit from targeted therapy a difficult challenge.

Multiplex ligation-dependent probe amplification is a new, high-resolution method for detecting numerous copy number variations in genomic sequences in a single reaction and requires only small amounts of DNA extracted from formalin-fixed paraffin-embedded tissues. ${ }^{16,17}$ This technique makes possible the simultaneous detection of the amplification status of ERBB2, EGFR, MET, MYC, $C C D 1$, and $M D M 2{ }^{16}$

The aims of the present study were to determine the gene amplification status of RTKs and other cell regulator genes including ERBB2, EGFR, FGFR2, $M E T, M Y C$, CCND1, and MDM2 in advanced gastric cancers with a view toward future introduction of molecular targeted therapy, and to examine the usefulness of multiplex ligation-dependent probe amplification in the semi-comprehensive detection of these gene amplifications.

\section{Materials and methods}

\section{Patients}

A total of 93 advanced gastric adenocarcinoma patients out of 262 gastric cancer patients who underwent surgery at the Department of Surgery in Kanazawa University Hospital between 2011 and 2013 were examined. Cancer staging was performed according to the TNM cancer staging system of the American Joint Committee of Cancer. ${ }^{18}$ The World Health Organization Classification of Tumors ${ }^{19}$ was used to determine histological classification as differentiated (tubular adenocarcinoma, papillary adenocarcinoma) or undifferentiated adenocarcinoma (mucinous adenocarcinoma, poorly cohesive carcinomas, mixed carcinoma, carcinoma with lymphoid stroma and unclassified carcinoma). This laboratory study was approved by the Medical Ethics Committee of Kanazawa University (Approval No 181), and written informed consent was obtained from all patients. Serial sections cut from representative formalin-fixed and paraffin-embedded cancer tissues were used for hematoxylin-eosin staining, multiplex ligation-dependent probe amplification, FISH, and immunohistochemistry.

\section{Multiplex Ligation-Dependent Probe Amplification}

A cancer area was selected on a $6 \mu$ m-thick representative tumor section, with reference to the adjacent hematoxylin-eosin staining section, taking care that non-neoplastic cells were excluded as much as possible. DNA was extracted manually from each section using proteinase $K$ (Rosch Diagnostics, Manheim, Germany) according to the manufacturer's (MRC-Holland, Amsterdam, The Netherlands) protocol. By using this protocol, $>1 \mu \mathrm{g}$ of sample DNA with an $\mathrm{OD}_{260}: \mathrm{OD}_{280}$ ratio within 1.1-1.7 was obtained from each tumor. DNA from the cell lines MKN7, A431, KATOIII, HSC39, and MKN45, which were previously shown to display amplified ERBB2, EGFR, FGFR2, FGFR2 and $M Y C$, and $M E T$, respectively, were used as positive controls. ${ }^{8,20}$

Multiplex ligation-dependent probe amplification analysis was performed by using two kits from MRCHolland. The SALSA MLPA KIT P175-A2 TumorGain kit contains two or three probes for each of 24 genes including ERBB2, EGFR, MET, MYC, CCND1, MDM2, and TOP2A. The SALSA MLPA probemix P231-A2 FGF10-FGFR2, which was originally used for the diagnosis of autosomal dominant lacrimoauriculodentodigital syndrome, ${ }^{21}$ contains five probes each for FGFR2 and FGF10. The PCR products were separated on an ABI-310 capillary sequencer (Applied Biosystems, Foster City, CA, USA) and interpreted with Genemapper software (Applied Biosystems). Data analysis was performed with Coffalyser MLPA-DAT software (version 9.4, MRCHolland) to generate normalized peak values. 


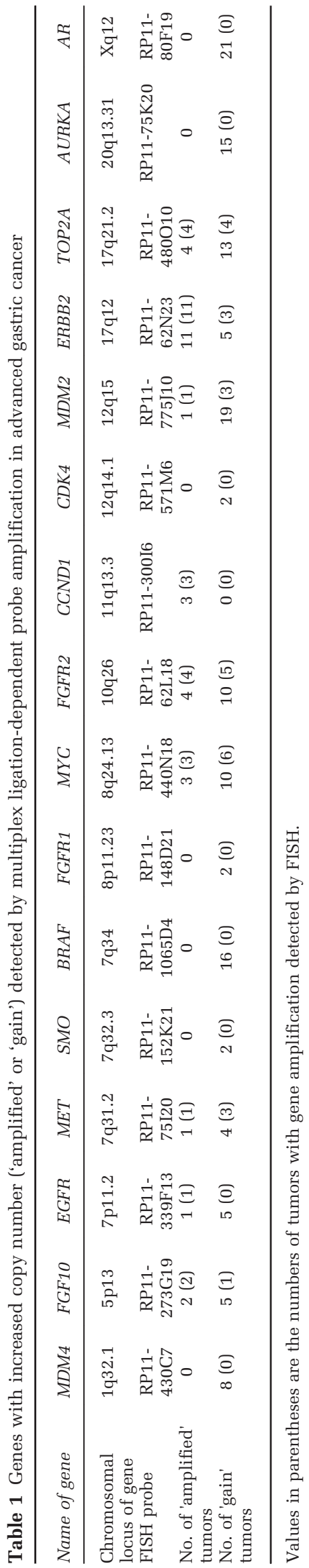

Average peak values below 0.7 were defined as 'lost', between 0.7 and 1.3 as 'normal', between 1.3 and 2.0 as 'gain', and $>2.0$ as 'amplified', as previously established. ${ }^{16,17}$

\section{FISH}

Tumors with 'amplified' or 'gain' of genes as determined by multiplex ligation-dependent probe amplification were further examined using FISH to validate the accuracy of the multiplex ligationdependent probe amplification results for the respective gene amplification. FISH probes acquired from BACPAC Resources (Oakland, CA, USA) are summarized in Table 1. The probes were labeled with SpectrumOrange or SpectrumGeen using a nick translation kit (Abbott Laboratories, Abbott Park, IL, USA). For the detection of gene amplification, a SpectrumGreen-labeled pericentromeric probe (Abbott), specific to each chromosome on which the particular gene was located, was co-hybridized to standardize the chromosome number. Tumors exhibiting co-amplification of different genes were further examined by simultaneous hybridization with two probes specific to the genes that were labeled with different fluorescent markers to determine the coexistence of the amplified genes in single cells and single amplicons.

Removal of protein from the tissue sections, denaturation, hybridization, and post-hybridization washing were performed as described previously. ${ }^{17}$ The tissue sections were counterstained with DAPI II (Abbott) and examined using a fluorescence microscope (Olympus, Tokyo, Japan) equipped with a Triple Bandpass Filter set (Abbott) for DAPI II, SpectrumOrange and SpectrumGreen, and a filter set specific for SpectrumOrange or SpectrumGreen. FISH images were recorded through a cooled chargecoupled device camera (DP-70, Olympus) linked to a computer software program (DP Manager, Olympus).

Scoring and evaluation of FISH slide were performed manually by counting gene signals and the centromere signals, on which the gene was located, of 20 tumor nuclei per case. The average copy number of the gene and the centromere, and their ratio, were determined for each case. Gene amplification was determined according to the American Society of Clinical Oncology/College of American Pathologists-approved criteria of ERBB2 amplification, except that the quantity of positive cells was defined as more than six gene copies per nucleus or gene signals/centromere signals $>2.2 .^{22}$ In addition, low-level amplification was arbitrarily defined as gene signals/centromere signals $>2.2$ and gene copy number of $<6$, and high polysomy was arbitrarily defined as gene signals/centromere signals $<1.8$ and gene copy number $>6$. In cases of apparent intratumoral heterogeneity in gene amplification, the fractions (\%) of the tumor cells containing gene 
Table 2 Antibodies and dilutions applied for immunohistochemistry

\begin{tabular}{llcll}
\hline Antibody & Clone & Dilution & Antigen retreval & Manufacturer \\
\hline ERBB2 & Polyclonal & $1: 400$ & None & Nichirei, Tokyo, Japan \\
EGFR & EGFR.25 & $1: 100$ & PC pH6, citrate & Novocastra Lab, Newcastle, UK \\
FGFR2 & ab58201 & $1: 1000$ & None & Abcam, Cambridge, UK \\
MYC & Y69 & $1: 50$ & PC pH9, EDTA & Pharmingen, San Diego, CA, USA \\
MDM2 & IF2 & $1: 40$ & PC pH6, citrate & Calbiochem, La Jolla, CA, USA \\
MET & SP44 & $1: 50$ & PC pH6, citrate & Spring Bioscience, Pleeasanton, CA, USA \\
CCND1 & SP4 & Prediluted & PC pH6, citrate & Nichirei, Tokyo, Japan \\
\hline
\end{tabular}

Abbreviation: PC, pressure cooking.

amplification were scored using broad tiers of $<30$, $30-70$, and $>70 \%$.

\section{Immunohistochemistry}

Immunohistochemistry for ERBB2, EGFR, FGFR2, MET, MYC, CCND1, and MDM2 was performed on tumors with FISH-proved amplification. The antibody clones, manufacturers and dilutions, and the antigen retrieval methods are summarized in Table 2. Antibody binding was visualized using the LSAB system (Dako, Glostrup, Denmark). For evaluation of positive staining of ERBB2, EGFR, FGFR2, and MET, each tumor, or portion of tumor, was scored using a four-tier system $(0,1+, 2+, 3+)$ according to the criteria recommended by Dako for the HercepTest, except that the quantity of positive cells was not considered. For evaluation of MYC, CCND1, and MDM staining, only nuclear immunostaining, which was significantly higher than that of control cells of normal gastric mucosa, was considered as positive.

\section{Statistical Analyses}

The association of gene amplification and histology of the tumor, and association between amplifications of different genes were analyzed for significance using either Fischer's exact probability test or the $\chi^{2}$-test.

\section{Results}

\section{Multiplex Ligation-Dependent Probe Amplification}

Multiplex ligation-dependent probe amplification analyses were successfully performed on all 93 of the formalin-fixed and paraffin-embedded advanced gastric adenocarcinoma tissue samples. The gene status of the 26 genes analyzed in the 93 tumors were categorized as 'amplified', 'gain', or 'normal' based on the mean multiplex ligation-dependent probe amplification peak values. There were no cases with 'lost' genes. The number of tumors with 'amplified' or 'gain' of specific genes by multiplex ligation-dependent probe amplification analysis is summarized in
Table 1. All tumors displayed a normal copy number of MYCN, ALK, PDGFRA, KIT, KDR, DHFR, ABL1, $R E T, C C N D 2$, and $A U R K B$.

\section{FISH}

Out of the total 93 advanced gastric cancers, 32 tumors $(34 \%)$ displayed gene amplification of one of FGFR10, EGFR, MET, MYC, FGFR2, CCND1, MDM2, $E R B B 2$, or TOP2A. In FISH analysis, most amplifications appeared as either tightly clustered or numerous scattered signals of the amplified genes, suggesting amplicons in homogeneously staining regions and double minute chromosomes, respectively. ${ }^{23}$ Cancer nuclei with colocalization of an amplified gene and the amplified centromeric lesion on which the gene is located, which results in a yellow fusion signal ${ }^{24}$ as shown in Supplementary Figure 1, was rarely observed. Approximately half of the gene amplifications occurred in an intratumoral heterogeneous fashion.

All tumors with genes that were categorized as 'amplified' by multiplex ligation-dependent probe amplification had cancer cells with gene amplification that could be detected by FISH as shown in Table 1. The tumors with 'gain' of MDM2, SMO, BRAF, FGFR1, CDK4, AURKA, and AR by multiplex ligation-dependent probe amplification showed polysomy of the chromosome on which the target gene is located; however, amplification of these genes was not found.

Table 3 describes the clinicopathological characteristics of the 32 tumors with gene amplification and indicates the fraction (\%) of the amplified cells and the type of amplification in each case. Three quarters (24 of 32) of the amplifications were of genes encoding RTKs: representative FISH images of amplified ERBB2, FGFR2, MET, and EGFR were shown in Figure 1a and b, Figures 1a and 2a, Figure 3a and c, and Figure 4a, respectively. Except for Case 7, amplification of these RTK genes occurred in a mutually exclusively manner, ie, each tumor had a single RTK gene mutation. In Case 7, dual-color FISH analysis demonstrated 'homogeneously staining region'-type ERBB2 amplification and 'double minute chromosome'-type FGFR2 amplification intermingled in a mosaic pattern. However, no single cell 


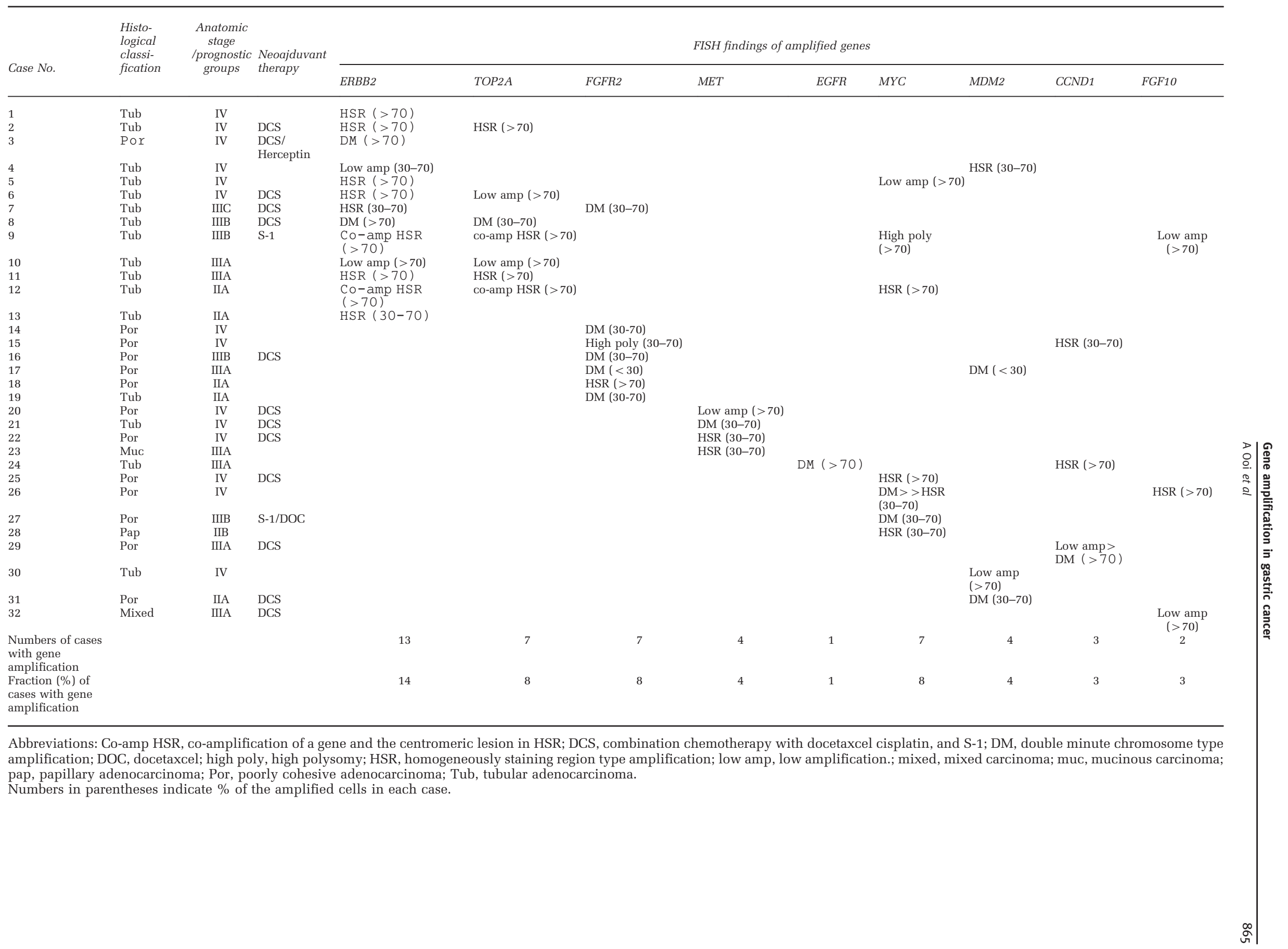


had amplification of both genes, as shown by dualcolor FISH in Figure 1a. MYC was amplified in seven tumors, and was co-amplified with ERBB2 in three tumors. On the basis of dual-color FISH analysis, coamplification of ERBB2 and $M Y C$ in single nuclei but on different amplicons was confirmed in case 9 (Figure 1b). MDM2 amplification was found in four tumors; one showed co-amplification with ERBB2 and the other showed co-amplification with the FGFR2; however, cancer cells with different gene amplification occupied different tumor areas as shown in Figure 2. CCND1 was amplified in three tumors; one showed co-amplification with EGFR and the other with FGFR2. Dual-color FISH analysis could confirm co-amplification of EGFR and CCND1 in single nuclei but on different amplicons in case 24 (Figure 4a). All of the tumors with TOP2A amplification were also positive for $E R B B 2$ amplification. Furthermore, dual-color FISH showed that TOP2A signals and ERBB2 signals were closely associated (Supplementary Figure 2).

Immunohistochemistry. Immunohistochemistry for ERBB2 indicated 11 tumors with $3+$ or $2+$ staining (Figure 1e), one tumor with 1+ staining (Case 7) (Figure 1c) and one tumor (case 10) was negative. Immunohistochemistry for FGFR2 displayed a variety of cytoplasmic staining from weak to strong, which was found to correspond to FGFR2-amplified tumor cells (Figures 1d and 2d). However, similar cytoplasmic staining was also commonly found in non-FGFR2-amplified cancer cells (Figure 2a, b, and d) and occasionally in non-neoplastic glandular cells. Immunohistochemistry of MET showed inconsistent results; $3+$ staining in Case 21 (Figure 3b), 2+ staining in Cases 22 and 23, and negative staining in Case 20. In Case 21, the MET amplification-positive and -negative cells were randomly intermingled; even single cancer tubules were composed of amplification-positive and -negative cells. FISH analysis of serial sections showed that the METamplified cells corresponded to MET 3+ immunostained cells, and that non-MET-amplified cells corresponded to MET immunohistochemicallynegative cells as shown in Figure 3a and b. In Case 22, MET-amplified and non-amplified cells were in different areas and both showed 2+ MET immunostaining. Thus these cells were not differentiated by immunohistochemistry as shown in Figure $3 e$ and $f$. Case 24 that showed homogeneous EGFR amplification showed $3+$ immunostaining of the EGFR (Figure 4b).

Most immunohistochemical results of MYC were equivocal (Figure 1f). In the immunohistochemistry analysis of MDM2 and CCND1, scattered positive nuclear staining was found and clear coincidence of this staining with the respective amplificationpositive nuclei was not confirmed (Figure 2c and e, Figure 4a and c, respectively).
Clinicopathological Findings. Amplification of $E R B B 2$ was significantly more frequent in differentiated carcinomas than in undifferentiated carcinomas $(P=0.04)$; however, the amplification of other genes showed no correlation with histological differentiation.

\section{Discussion}

In the present study, 32 of a total of 93 advanced gastric cancers (34\%) displayed definite gene amplification and most of this amplification occurred in a FISH pattern that was reminiscent of cytogenetic homogeneously staining region or double minute chromosomes. Furthermore, 26\% (24 of 93) of the amplifications were amplification of one of four genes (ERBB2, FGFR2, MET, and EGFR) that encode a RTK. The frequencies of these gene amplifications were not significantly different from those previously reported for ERBB2, FGFR2,9,10,12,14 $M E T{ }^{6,7,14,15}$ and $E G F R{ }^{6,20}$ It is intriguing that, except for case 7 , RTK gene amplification occurred in a mutually exclusive manner. Even in Case 7, RTK gene co-amplification did not occur in the same cells. Thus, if a common tyrosine kinase inhibitor to the four RTKs were available, 24 of 93 (26\%) cases of advanced gastric cancer would potentially be susceptible to that agent. This characteristic of RTKs is one of the reasons why RTK gene amplifications have attracted much attention as molecular targets even though the prevalence of individual RTK gene amplification is low. $6,12,13$

However, if small fractions of amplified cells that cannot be detect by screening with multiplex ligation-dependent probe amplification and/or early gastric cancers are included, co-amplification of RTK genes is not exceptional in gastric cancers. On the contrary, our previous study showed a marginal trend for co-amplification of EGFR and ERBB2; however, this trend was not statistically significant. ${ }^{25}$ In our more recent study in which we examined the co-amplification of ERBB2 and other RTK genes, we found that of 51 tumors with $E R B B 2$ amplification, $14 \%(7 / 51)$ of the tumors displayed co-amplification with EGFR, one tumor showed coamplification with FGFR2, and one tumor showed co-amplification with MET and FGFR2. ${ }^{16}$ It is probable that redundancy of RTKs, which are unnecessary for cancer cell growth, diminishes in the course of gastric cancer progression.

In contrast to the lack of co-amplification of RTK genes, MYC, and RTK genes are occasionally coamplified in single cancer cells. In the present study co-amplification of $M Y C$ and ERBB2 in the same cell was found in three tumors, and, in our previous study, co-amplification of $M Y C$ and ERBB2 or EGFR occurs in a non-incidental manner. ${ }^{25}$ Thus, these coamplifications may be of some merit for survival of cancer cells. A similar selective co-amplification of ERBB2 and MYC was found in breast cancer cells 

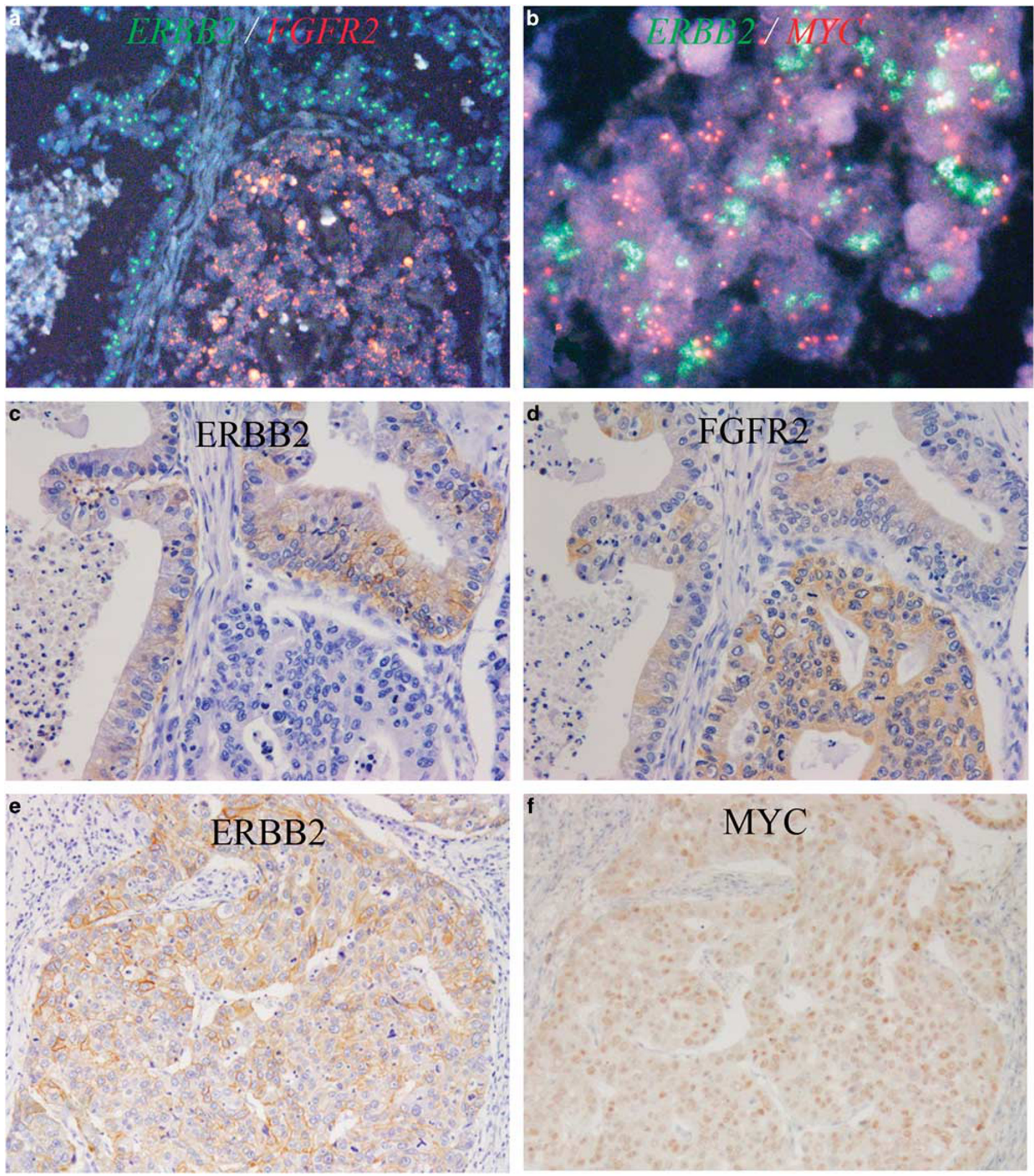

Figure 1 Gastric cancers with co-amplification of ERBB2 and FGFR2 (Case 7), and ERBB2 and MYC (Case 9). In Case 7, amplification of ERBB2 and FGFR2 was found in different cancer cells. In Case 9, ERBB2 and MYC was in the same nuclei. Gene amplifications were analyzed by dual-color FISH (a: green fluorescence, ERBB2; orange fluorescence, FGFR2) (b: green fluorescence, ERBB2; orange fluorescence, $M Y C$ ) and their protein overexpression was analyzed by immunohistochemistry (c and ee, ERBB2; d, FGFR2; f, MYC). Panels a, c, and $\mathbf{d}$ show the same field. ERBB2- and FGFR2-amplified cells in panel a correspond to the 1+ ERBB2-stained cells in panel $\mathbf{c}$ and the cells with weak cytoplasmic staining of FGFR2 in panel d, respectively. The cells with gene amplification in panel b showed $2+$ immunostaining of ERBB2 and equivocal nuclear staining of MYC. 

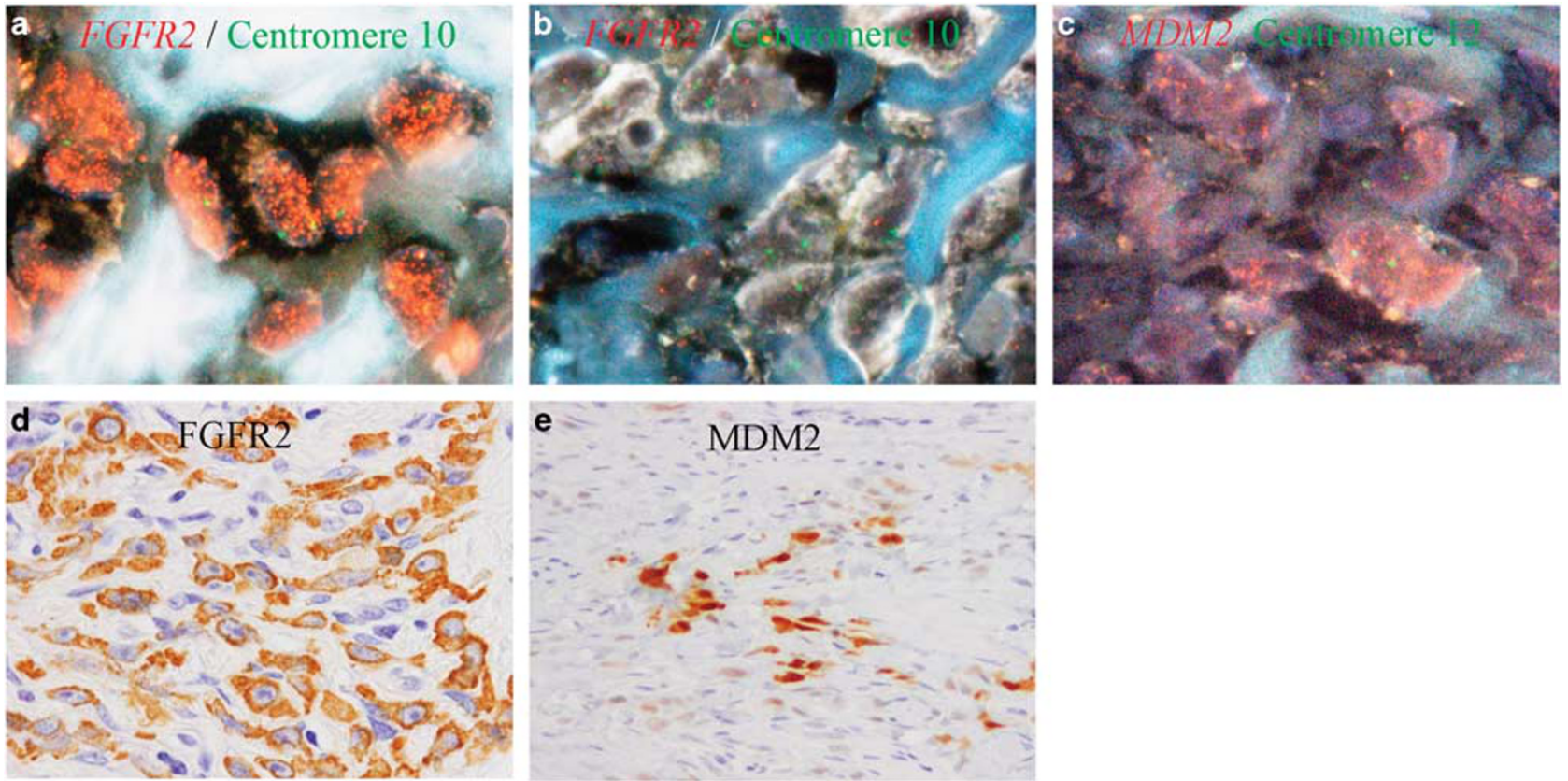

Figure 2 A poorly cohesive-type gastric cancer with FGFR2-amplified and MDM2-amplified cancer cells in different areas (Case 17). FGFR2 and MDM2 gene amplification was analyzed by dual-color FISH (a and b: orange fluorescence, FGFR2; green fluorescence, centromere 10. c: orange fluorescence, $M D M 2$; green fluorescence, centromere 12) and their protein overexpression was analyzed by immunohistochemistry (d, FGFR2; E, MDM2). Panels a and b are different fields of the same area of panel d. Intense cytoplasmic staining of FGFR2 (d) was detected in both FGFR2-amplified (a) and FGFR2-non-amplified (b) cells. Panels $\mathbf{c}$ and e are the same area. Most but not all MDM2-amplified cells show 3+-nuclear staining of MDM2.

and it has been speculated that this co-amplification might be a very favorable prognostic factor when combined with adjuvant therapy by trastuzumab. ${ }^{26,27}$ However, this hypothesis was recently refuted. Instead, new data have shown that this coamplification is associated with poor outcome and is associated with the sequence of acquisition of a malignant stem cell-like phenotype that maintains lower growth and therefore may be a key target of new therapy. ${ }^{28}$

TOP2A resides very close to ERBB2 on the chromosome and its amplification was only observed with the concomitant amplification of ERBB2. However, TOP2A is not simply a 'passenger' gene because TOP2A encodes an enzyme, topoisomerase II $\alpha$, which is required for DNA replication. Moreover, clinical studies have confirmed that breast cancer patients with TOP2A gene amplification are more sensitive to topoisomerase II $\alpha$-based therapy. ${ }^{29}$ Unfortunately this therapy is not commonly used for the treatment of gastric cancers in Japan.

Information regarding $C C N D 1$ amplification is useful because expression of CCND1 may cause resistance to cisplatin, ${ }^{30}$ which was coadministered with trastuzumab in the ToGA clinical trial. To the best of my knowledge, this is the first report of amplification of FGF10 in gastric cancer. Although stomach development is dependent on FGF10- and FGFR2-mediated signaling, ${ }^{31}$ it remains to be clarified whether FGF10 amplification is relevant to carcinogenesis of gastric cancer through enhanced signaling. In this study an additional candidate for molecular targeting, amplified $M D M 2$, was found in ERBB2-non-amplified gastric cancers. MDM2 is an oncogene whose expression controls tumorigenesis by promoting degradation of the tumor suppressor protein p53. Amplification of MDM2 impacts cancer treatments that use selective inhibitors of MDM2 proteins. ${ }^{32,33}$ Indeed, in an in vivo study, treatment with nutlin, an MDM2 antagonist, induced p53-dependent transcription and apoptosis in liposarcoma cells that were positive for MDM2 amplification. ${ }^{34}$

In this study we screened advanced gastric carcinomas for possible gene amplification by multiplex ligation-dependent probe amplification and confirmed the results obtained using FISH analysis, which has been considered as the gold standard method for analysis of gene amplification. FISH probes span $\sim 200 \mathrm{~kb}$ of DNA sequence, which is similar to or several-fold larger than the target gene size. In contrast, multiplex ligation-dependent probe amplification targets intragenic portions of DNA sequence with sequence sizes of $50-100$ bases. Although for a typical gene amplification the result of multiplex ligation-dependent probe amplification should theoretically coincide with that of FISH, the copy number detected by multiplex ligationdependent probe amplification was often lower than that detected by FISH owing to intratumoral heterogeneity and/or contamination of non-neoplastic DNA. In the present study, the cutoff value of 1.3 that was recommended by the multiplex ligationdependent probe amplification kit manufacturer was 

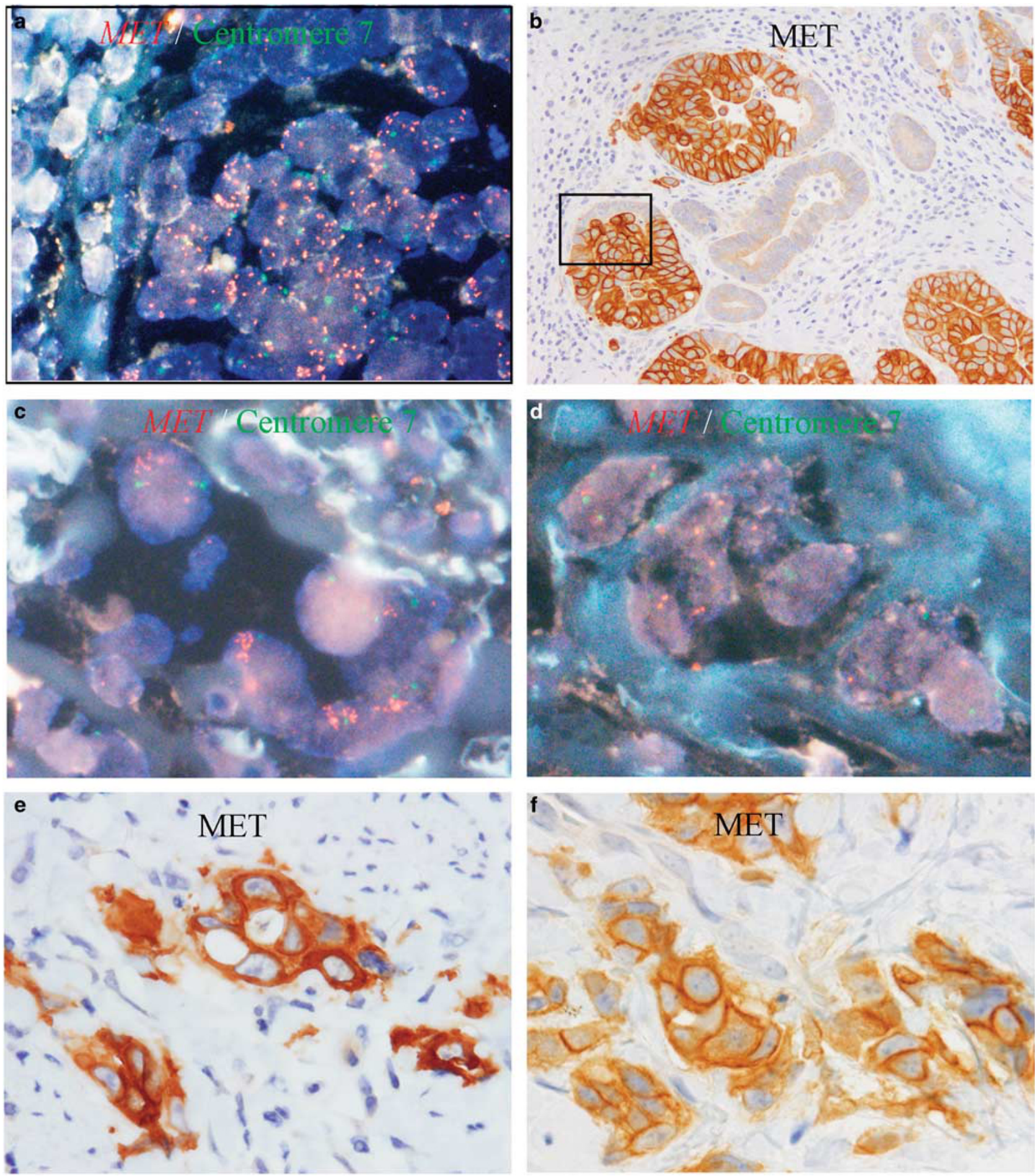

Figure 3 Amplification and overexpression of MET in gastric carcinomas (Cases 21 and 22). A tubular adenocarcinoma (Case 21, a and b) and a poorly cohesive-type adenocarcinoma (Case 22, $\mathbf{c}-\mathbf{f}$ ) were analyzed for MET gene amplification by dual-color FISH (a, c, and d: orange signals, MET; green signals, centromere 7) and for MET protein overexpression by immunohistochemistry (b, e, and f). In the tubular adenocarcinoma (Case 21), cancer cells with 3+-immunostaining of MET displayed amplified MET; cells with negative immmunostaining displayed no amplification (a and $\mathbf{b}$ ). The region enclosed by the black rectangle in panel $\mathbf{b}$ corresponds to the region shown in panel a. The poorly cohesive type adenocarcinoma (Case 22) consisted of MET-amplified (c) and non-amplified cancer cells (d) in different areas, and both areas displayed 2+-staining for MET by immunohistochemistry (e and f). Panels $\mathbf{c}$ and $\mathbf{e}$, and panels $\mathbf{d}$ and $\mathbf{f}$ are the same field. 

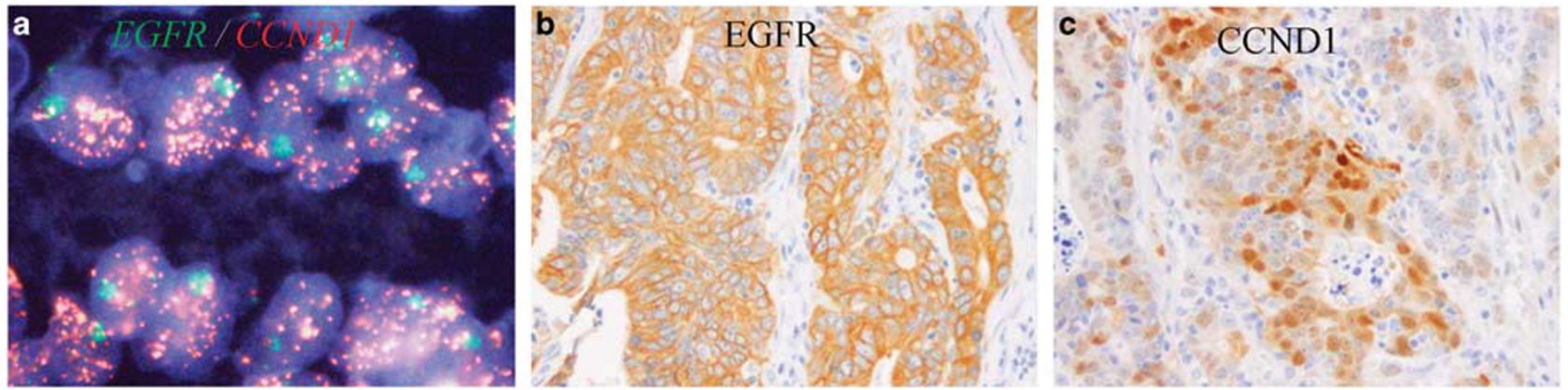

Figure 4 A gastric cancer with co-amplification of EGFR and CCND1 in the same nuclei (Case 24). EGFR and CCND1 gene amplification was analyzed by dual-color FISH (a: green fluorescence, EGFR; orange fluorescence, CCND1), and their protein overexpression was analyzed by immunohistochemistry (b, EGFR; c, CCND1). The gene-amplified cells showed 3+-immunostaining of the EGFR and scattered nuclear staining of CCND1.

used for all of the genes. Although all of the cases defined as 'amplified' by multiplex ligationdependent probe amplification were also amplified by FISH analysis, seven genes that were defined as 'gain' by multiplex ligation-dependent probe amplification showed no real amplification by FISH analysis (Table 1). It may therefore be possible to increase the specificity of the test by resetting the cutoff values for these genes. ${ }^{35}$ However, for the purpose of screening of gene amplification, high sensitivity precedes specificity and the cutoff value of 1.3 was very acceptable for such screening.

$M Y C, C C N D 1, M D M 2, E R B B 2, E G F R, F G F R 2$, and $M E T$ are considered to be involved in carcinogenesis through protein overexpression of amplified genes. Thus, immunohistochemistry that is familiar to most diagnostic pathologists might be the first choice for examination of these aberrations. However, our attempts to correlate FISH status with immunohistochemical staining resulted in variable correlations between the two types of measurement ranging from complete correspondence to dissociation. The protein overexpression of $M Y C, C C N D 1$, and $M D M 2$ is regulated by several pathways and thus gene amplification may not necessarily provide a straightforward correlation with protein overexpression. As for RTK genes, the hypothesis that 'amplification causes overexpression' is fundamentally established for ERBB2, and is highly supported for EGFR, FGFR2, and MET. ${ }^{10-14}$ However, even for RTKs, inconsistent results between FISH and immunohistochemical analyses were often obtained, mostly because of inherent technical reasons of immunohistochemistry such as specificity of antibodies, fixative used, duration of fixation, staining procedures, and/or scoring system. Therefore, at the present time gene amplification provides the best biomarkers for helping to select the right patient candidate for molecular therapy targeted toward these genes, and multiplex ligation-dependent probe amplification is the most feasible method for screening these gene amplifications.

In conclusion, given the apparent low prevalence of amplification of candidate target oncogenes for therapy of gastric cancer, implementation of semi-comprehensive screening by multiplex ligation-dependent probe amplification followed by confirmatory FISH analysis should facilitate the identification of patients who may benefit from such tailored therapy.

\section{Acknowledgments}

This work was financially supported by the Japanese Ministry of Education, Sports, Science, and Culture (C2546015 (SF), C25460452 (AO), C26460438 (YD), and Young Scientists (B) (25860266) (TO) and (26860235) (RN)), and Smoking Research Foundation (YD).

\section{Disclosure/conflict of interest}

The authors declare no conflict of interest.

\section{References}

1 Jemal A, Bray F, Center MM, et al. Global cancer statistics CA. Cancer J Clin 2011;61:69-90.

2 Japanese Research Society for Gastric Cancer The general rules for the gastric canceer study in surgery and pathology: Part I-Clinical classification. Jpn J Surg 1981;11:127-139.

3 Nashimoto A, Akazawa K, Isobe Y, et al. Gastric cancer treated in 2002 in Japan: 2009 annual report of the JGCA nationwide registry. Gastric Cancer 2013;16: $1-27$.

4 Bang YJ, Van Cutsem E, Feyereislova A, et al. Trastuzumab in combination with chemotherapy versus chemotherapy alone for treatment of HER2-positive advanced gastric or gastro-oesophageal junction cancer (ToGA): a phase 3, open-label, randomised controlled trial. Lancet 2010;376:687-697.

5 Lee J, Ou SH. Towards the goal of personalized medicine in gastric cancer-time to move beyond HER2 inhibition. Part II: Targeting gene mutations and gene amplifications and the angiogenesis pathway. Discov Med 2013;16:7-14.

6 Lennerz JK, Kwak EL, Ackerman A, et al. MET amplification identifies a small and aggressive subgroup of esophagogastric adenocarcinoma with evidence of 
responsiveness to crizotinib. J Clin Oncol 2011;29: 4803-4810.

7 An X, Wang F, Shao Q, et al. MET amplification is not rare and predicts unfavorable clinical outcomes in patients with recurrent/metastatic gastric cancer after chemotherapy. Cancer 2014;120:675-682.

8 Hara T, Ooi A, Kobayashi M, et al. Amplification of c-myc, K-sam, and c-met in gastric cancers: detection by fluorescence in situ hybridization. Lab Invest 1998;78:1143-1153.

9 Jung EJ, Jung EJ, Min SY, et al. Fibroblast growth factor receptor 2 gene amplification status and its clinicopathologic significance in gastric carcinoma. Hum Pathol 2012;43:1559-1566.

10 Matsumoto K, Arao T, Hamaguchi T, et al. FGFR2 gene amplification and clinicopathological features in gastric cancer. Br J Cancer 2012;106:727-732.

11 Smolen GA, Sordella R, Muir B, et al. Amplification of MET may identify a subset of cancers with extreme sensitivity to the selective tyrosine kinase inhibitor PHA-665752. Proc Natl Acad Sci USA 2006;103: 2316-2321.

12 Xie L, Su X, Zhang L, et al. FGFR2 gene amplification in gastric cancer predicts sensitivity to the selective FGFR inhibitor AZD4547. Clin Cancer Res 2013;19: 2572-2583.

13 Deng N, Goh LK, Wang H, et al. A comprehensive survey of genomic alterations in gastric cancer reveals systematic patterns of molecular exclusivity and cooccurrence among distinct therapeutic targets. Gut 2012;1:73-684.

14 Liu YJ, Shen D, Yin X, et al. HER2, MET and FGFR2 oncogenic driver alterations define distinct molecular segments for targeted therapies in gastric carcinoma. Br J Cancer 2014;110:1169-1178.

15 Lee HE, Kim MA, Lee HS, et al. MET in gastric carcinomas: comparison between protein expression and gene copy number and impact on clinical outcome. Br J Cancer 2012;107:325-333.

16 Tajiri R, Ooi A, Fujimura T, et al. Intratumoral heterogeneous amplification of ERBB2 and subclonal genetic diversity in gastric cancers revealed by multiple ligationdependent probe amplification and fluorescence in situ hybridization. Hum Pathol 2014;45:725-734.

17 Ooi A, Inokuchi M, Harada S, et al. Gene amplification of ESR1 in breast cancers-fact or fiction? A fluorescence in situ hybridization and multiplex ligation-dependent probe amplification study. J Pathol 2012;227:8-16.

18 Flemming ID, Cooper IS, Henson DE, et al. Stomach. In: AJCC Cancer Staging Manual 5th edn. Ch. 10. Lippincott Williams and Wilkins: Philadelphia, PA, USA, 1997, pp 71-76.

19 Fenoglio-Preiser C. Tumours of the stomach. In: Hamilton S, Aaltonen SA (eds). WHO classification of tumours. IARC Press: Lyon, France, 2000, pp 37-52.

20 Takehana T, Kunitomo K, Suzuki S, et al. Expression of epidermal growth factor receptor in gastric carcinomas. Clin Gastroenterol Hepatol 2003;1:438-445.

21 Rohmann E, Brunner HG, Kayserili H, et al. Mutations in different components of FGF signaling in LADD syndrome. Nature Genet 2006;38:414-417.
22 Wolff AC, Hammond ME, Schwartz JN, et al. American Society of Clinical Oncology/College of American Pathologists guideline recommendations for human epidermal growth factor receptor 2 testing in breast cancer. J Clin Oncol 2007;25:118-145.

23 Kobayashi M, Ooi A, Oda Y, et al. Protein overexpression and gene amplification of c-erbB-2 in breast carcinomas: a comparative study of immunohistochemistry and fluorescence in situ hybridization of formalin-fixed, paraffin-embedded tissues. Hum Pathol 2002;33:21-28.

24 Starczynski J, Atkey N, Connelly Y, et al. HER2 gene amplification in breast cancer: a rogues' gallery of challenging diagnostic cases: UKNEQAS interpretation guidelines and research recommendations. Am J Clin Pathol 2012;137:595-605.

25 Mitsui F, Dobashi Y, Imoto I, et al. Non-incidental coamplification of Myc and ERBB2, and Myc and EGFR, in gastric adenocarcinomas. Mod Pathol 2007;20:622-631.

26 Kim C. Trastuzumab sensitivity of breast cancer with co-amplification of HER2 and cMyc in vivo. Breast Cancer Res Treat 2005;54(Suppl 1):S6(abstract).

27 Perez EA, Jenkins RB, Dueck AC, et al. C-MYC alterations and association with patient outcome in early-stage HER2-positive breast cancer from the north central cancer treatment group N9831 adjuvant trastuzumab trial. J Clin Oncol 2011;29:651-659.

28 Nair R, Roden DL, Teo WS, et al. c-Myc and Her2 cooperate to drive a stem-like phenotype with poor prognosis in breast cancer. Oncogene 2013;33: 3992-4002.

29 Di Leo A, Gancberg D, Larsimont D, et al. HER-2 amplification and topoisomerase Ilalpha gene aberrations as predictive markers in node-positive breast cancer patients randomly treated either with an anthracycline-based therapy or with cyclophosphamide, methotrexate, and 5-fluorouracil. Clin Cancer Res 2002;8:1107-1116.

30 Noel EE, Yeste-Velasco M, Mao X, et al. The association of CCND1 overexpression and cisplatin resistance in testicular germ cell tumors and other cancers. Am J Pathol 2010;176:2607-2615.

31 Spencer-Dene B, Sala FG, Bellusci S, et al. Stomach development is dependent on fibroblast growth factor 10/fibroblast growth factor receptor 2b-mediated signaling. Gastroenterology 2006;130:1233-1244.

32 McInnes C, Wang S, Anderson S, et al. Structural determinants of CDK4 inhibition and design of selective ATP competitive inhibitors. Chem Biol 2004;11: 525-534.

33 Vassilev LT, Vu BT, Graves B, et al. In vivo activation of the p53 pathway by small-molecule antagonists of MDM2. Science 2004;303:844-848.

34 Muller CR, Paulsen EB, Noordhuis P, et al. Potential for treatment of liposarcomas with the MDM2 antagonist Nutlin-3 A. Int J Cancer 2007;121:199-205.

35 Moelans CB, de Weger RA, van Diest PJ. Multiplex ligation-dependent probe amplification to detect HER2 amplification in breast cancer: new insights in optimal cut-off value. Cell Oncol 2012;32:311-312.

Supplementary Information accompanies the paper on Modern Pathology website (http://www.nature.com/ modpathol) 